stretched positions, and it is thus that the artist should represent them. It is his duty to represent things as they appear, rather than as they actually are, at a given instant of time.

The fan-shaped form noticed in the case of the oscillating pencil becomes exceedingly indistinct, if it does not disappear altogether, in the case of the galloping horse's legs. This is owing to the rapid internal changes of form of the legs.

Your correspondent, Sir W. G. Simpson, Bart., states in his excellent letter produced in NATURE, vol.xxi. p. 55, that a galloping horse might be represented with all its legs gathered under it. I venture to disagree with him for this reason: the two "minimums" to which I have referred in a former part of this letter are not coincident when the legs are in their extreme position gathered under the body, and therefore no such distinct image of them in that position is produced. The "minimums" are only coincident in the other extreme, viz., the outstretched, position.

The artistic representation of a horse's paces other than galloping, as also that of other objects in motion, can be determined by similar reasoning. V. B. BARRINGTON-KENNETT

15, Hyde Park Gardens, W., November 26

\section{Force and Momentum}

IT is commonly said that change of momentum is evidence that force has acted or is acting on the mass, and that the rate at which the momentum is changing is the measure of the force. Thus, in his lecture on "Force," Prof. Tait says : "Force is the rate of change of momentum" (NATURE, vol. xiv. p. 462). This is not true if the mass be variable. Suppose a sphere of ice moving with constant velocity in a straight line through hot space. The mass, and therefore the momentum, is changing at every instant by the evaporation of the ice. The evaporation being supposed uniform over the entire surface, any force impressed on the sphere by the mutual repulsion between it and a particle of vapour thrown off at a point, $\not$, is balanced by an equal force at the other end of the diameter through $p$. Hence, the resultant of all these forces is nothing. Here, then, we have change of momentum of the sphere, although no force acts on it. In like manner the change of momentum of a rocket and of a locomotive engine is partly due to change of mass. Does it not hence appear that change of velocity is the proper evidence of the action of force? When a variable mass, $m$, is in motion, the proper measure of the force acting on $m$ at any given instant in any given direction is - not the rate of change of momentum, but-the product of the value of the mass at that instant, and the value of the rate of change of the velocity at that instant and in that direction, i.e., the measure of the force is not $\frac{d(m v)}{d t}$, but $m \frac{d v}{d t}$.

E. G.

[There is no such thing in nature as a "variable mass"; and our correspondent's difficulty arises from his omitting to take account of the momentum of each of the parts (however small) into which a mass may be divided. In most good works on dynamics he will find the motion of a rocket, or of a descending rain-drop (which gathers mass as it falls), accurately treated on the assumption that the momentum produced per unit of time is the measure of the force acting.-ED.]

Change in Apparent Position of Geometrical Figures

THE perplexing illusion to which $\mathrm{Mr}$. Bellamy refers (NATURE, vol. xx. p. 362) has long been known, and various explanations have been given of it by physicists. Sir Chas. Wheatstone, in 1838, showed clearly that it is a mental operation, while combating the idea of Prof. Necker, of Geneva, who attri-

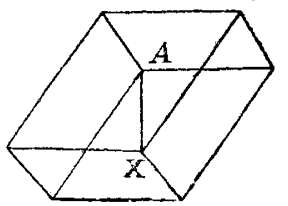

buted the alteration of appearance in geometrical figures, not to a mental operation, but to an involuntary change in the adjustment of the eye for obtaining distinct vision. Necker's experiment is substantially the same as that described by Mr. Bellamy. The solid angles at $\mathrm{A}$ and $\mathrm{X}$ being alternately looked at, sometimes one and sometimes the other appears the nearer, the entire figure at the same time changing in unison; and as Wheatstone points out, "the change of figure frequently occurs while the eye continues to look at the same angle."

In the following experiment it is seen more clearly still that the operation is a mental one, because there is neither movement of recti, oblique, nor ciliary muscles. Two concentric squares have their corners joined by straight lines. The lesser square

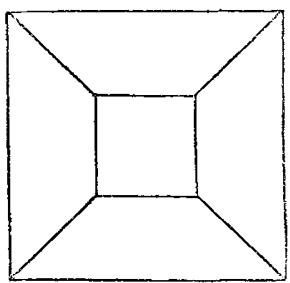

will appear in a plane anterior, or posterior to the larger, according as we regard the figure as the representation of a truncated pyramid, or as the representation of a room with its sides all sloping away to the distant square wall. Here no eye muscles are concerned; the image on the retina remains unaltered, and the only operation is a mental one, a turning to the results of past experience.

WM. ACKROYD

\section{Mutual Attraction of Spectral Lines}

I Do not know that it has been remarked that a line in the diffraction-spectrum (whether bright or dark) must be shifted from its normal position in case another line falls very near it. Neighbouring lines must be attracted if both are bright or both dark, and repelled if one is bright and the other dark. The reason is that the lines are only maxima or minima of light, and the differential coefficient of the sum does not vanish at the same points as the differential coefficients of the separate terms. The shifting will be the greatest in the case of a faint line near a very intense one. I have succeeded in this way in shifting the positions of lines by measurable amounts ( $x^{\prime \prime}$ to $2^{\prime \prime}$ ).

Baltimore, Md., November I4

\section{S. Peirce}

\section{EXPLORATION OF TIMOR}

$\mathrm{T} T$ will be perhaps of some interest to the readers of 1 NATURE to hear that Mr. Riedel, the Dutch resident on Timor (Timor Kupang), who formerly lived on Celebes, and collected a great deal on this island for European museums, and who is known by his various writings on different scientific questions concerning the East, has just returned from a twenty-five days' journey through Central Timor from $123^{\circ} 30^{\prime}-125^{\circ}$ E.L., as he wrote to me in a letter dated October 6. No European has made such a journey through Timor before, and it has been very troublesome. But the country is, Mr. Riedel remarks, a splendid one, and very suitable for coffee and cinchona cultivation. The traveller did not see any Negritos, who, according to the assertion of M. Hamy, live in the interior of Timor, nor did he hear anything of a Casuary which was reported from there recently. Mr. Riedel collected many geographical notes, and sketched a map of the parts which he visited. A small collection of plants was forwarded to me by $\mathrm{Mr}$. Riedel, and I have sent them to Kew, as Prof. Oliver formerly had the kindness to determine several botanical collections of Mr. Riedel's from Celebes.

Dresden, November 29,

Royal Zool. Museum

LAND SHELLS OF THE AUSTRAL ISLANDS

THE small island of Rurutu (Oheatora of Capt. Cook) is about 320 miles south-south-west of Tahiti; it is eight miles in length, and has an elevation of I, 500 feet, over 100 feet consisting of old coral reefs which have been upheaved to that altitude. Mr. Charles de Gage, a resident and experienced naturalist, has collected a number of land-shells, which have been studied 